\title{
A comparison of two approaches to managing acute primary angle closure in Asian eyes
}

This article was published in the following Dove Press journal:

Clinical Ophthalmology

17 June 2013

Number of times this article has been viewed

\author{
Henrietta Ho' \\ Paul T Chew ${ }^{2}$ \\ Chelvin Sng ${ }^{1,2}$ \\ Huiqi Huang' \\ Tin Aung ${ }^{1,2}$ \\ Shamira A Perera ${ }^{1,2}$ \\ 'Singapore Eye Research Institute, \\ Singapore National Eye Centre, \\ Singapore; ${ }^{2}$ Yong Loo Lin School \\ of Medicine, National University \\ of Singapore and National University \\ Health System, Singapore
}

Purpose: To review the management regimes of acute primary angle closure (APAC) in two hospitals in Singapore, and to identify the incidence of and risk factors for progression to glaucomatous optic neuropathy.

Methods: We conducted a retrospective review of 40 patients from National University Hospital (NUH) and 52 patients from Singapore National Eye Centre (SNEC) who were diagnosed with APAC. Patients were treated with similar protocols of intensive medical therapy until laser peripheral iridotomy could be performed. In the event of failed medical treatment, patients at NUH only underwent laser iridoplasty. The 1-year outcomes were reviewed.

Results: The demographic features of patients and presenting intraocular pressures (IOP) were similar in both centers. More patients from NUH presented within 3 days of symptom onset, compared to those from SNEC $(90.0 \%$ versus $71.2 \%$, respectively) $(P=0.037)$. The mean \pm standard deviation time to break the attack was $18.2 \pm 32.9$ hours at SNEC and 9.80 \pm 10.6 hours at NUH $(P=0.11)$. The mean follow up duration was $18.8 \pm 14.0$ months. Nineteen patients (36.5\%) from SNEC and six patients $(22.5 \%)$ from NUH developed raised IOP $(P=0.032)$ within 1-year of the attack. Of these, glaucomatous optic neuropathy developed in thirteen patients $(68.4 \%)$ from SNEC and all six patients (100\%) from NUH. At final review, the mean IOP of the APAC eye was $14.8 \pm 4.3 \mathrm{mmHg}$ from SNEC and $13.4 \pm 3.0 \mathrm{mmHg}$ from NUH. There was no significant difference in final visual acuity or IOP between both groups.

Conclusion: Treatment strategies in both centers were effective in aborting an APAC attack. The development of raised IOP appears to be associated with a longer period of attack suggesting that greater urgency in aborting APAC attacks may entail better long term outcomes.

Keywords: acute primary angle closure, management, primary angle closure glaucoma

\section{Introduction}

Acute primary angle closure (APAC) is a potentially blinding ocular emergency. Studies have shown that a single attack has been associated with permanent retinal nerve fiber layer loss, visual field defects, and optic disc pallor. ${ }^{1-3}$ Despite Singapore having the highest reported incidence of APAC of any country studied to date, consensus on an ideal management protocol has yet to be devised for its ophthalmic centers. ${ }^{4}$

Previous studies conducted at different centers in Singapore revealed contradictory evidence for the risk of progression to primary angle closure glaucoma (PACG) following prior APAC after laser peripheral iridotomy (LPI). An initial study conducted at the National University Hospital (NUH) between 1990 and 1994 revealed that a majority (58.1\%) of these Asian eyes required additional treatment with ocular hypotensive medication, and $32.7 \%$ of patients required filtration surgery after an APAC episode. 5 A decade later, Tan et al reported the long term outcomes of APAC patients at the same
Correspondence: Shamira Perera Singapore National Eye Centre, II Third Hospital Avenue, I6875I Singapore

Tel +6562277255

Fax +65 62263395

Email shamiraperera@hotmail.com 
center and showed that only $21.4 \%$ of patients who presented between December 2003 and June 2006 developed chronically raised intraocular pressures post APAC. ${ }^{6}$ This difference was attributed to a shorter duration of symptoms and earlier time to presentation for treatment in the latter population.

Conventionally, treatment of APAC involves the use of topical and systemic medication to reduce intraocular pressure (IOP), and definitive LPI is delayed until there is sufficient corneal clarity to be performed safely. ${ }^{7}$ In patients not suitable for immediate definitive LPI, argon laser peripheral iridoplasty (ALPI) has been reported to be significantly more effective compared to medical therapy in reducing IOP during APAC, and this was independent of the duration of attack. ${ }^{8}$

There is conflicting evidence as to whether a delay in aborting an acute attack carries a significant risk of developing glaucomatous optic neuropathy (GON). ${ }^{9-11}$ Cumulating evidence promotes a more rapid approach to IOP lowering in APAC, yet the role of alternative management protocols is inadequately answered.

Within Singapore, the NUH advocates conventional medical treatment and ALPI is performed if the attack is not broken within an hour. Conversely, at another center, the Singapore National Eye Centre (SNEC), topical and intravenous drugs are used as the primary therapy, and ALPI is extremely rarely implemented. The duration of the attack and outcomes at 1-year in patients at both centers are expected to be different.

In this study, we aimed to compare the two approaches in Singapore regarding the incidence of progression to GON, and to identify risk factors predicting progression to GON.

\section{Methods}

This was a retrospective cohort study of consecutive patients who were diagnosed with APAC at two hospitals in Singapore: SNEC and NUH. At NUH, 40 consecutive patients who presented with APAC between December 2003 and June 2006 were included, as previously described. ${ }^{6}$ At SNEC, the charts of 52 consecutive patients who presented between June 2010 and November 2011 were reviewed. Patients were identified from a logbook of acute cases in the emergency department as well as from the database of another ongoing APAC trial at the center. The study protocol had the approval of the institutional review board of the respective clinical ethics committees, and was conducted in accordance to the tenets of the Declaration of Helsinki.

The criteria used to define APAC are as follows: (1) At least two of these symptoms: ocular or periocular pain, nausea and/or vomiting, an antecedent history of intermittent blurring of vision with haloes around lights; (2) The presenting IOP of $>21 \mathrm{mmHg}$, as measured by Goldmann applanation tonometry, with the presence of at least three of the following signs: conjunctival injection, corneal epithelial edema, a mid-dilated unreactive pupil and a shallow anterior chamber with iridotrabecular contact.

Following APAC resolution, the development of PACG was defined as eyes with IOP $>21 \mathrm{mmHg}$ measured by Goldmann applanation tonometry without the use of ocular hypotensive medication associated with glaucomatous optic neuropathy (defined as loss of neuroretinal rim with a vertical cup:disc ratio of $\geq 0.6$, and/or notching attributable to glaucoma) and reproducible visual field loss compatible with glaucoma on static white-on-white threshold perimetry (program 2402 SITA, Humphrey Instruments, Dublin, CA, USA); that is, glaucoma hemifield test results outside normal limits and/or an abnormal pattern standard deviation with $P<0.05$ occurring in the normal population. ${ }^{6}$ Reliability criteria for perimetry used were fixation losses $<20 \%$, false positive and false negative of $<33 \%$ each, and the presence of the visual field defect in at least two reliable visual field tests. ${ }^{6}$

Patients who were diagnosed with secondary angle closure, such as from peripheral anterior synechiae in neovascular glaucoma, and those with evidence of primary angle closure glaucoma in the APAC eye at presentation, were excluded. The presenting features at the time of diagnosis, such as initial visual acuity (VA) and initial IOP, acute treatment and subsequent management, as well as outcomes at 1-year were analyzed.

Initial treatment for APAC at $\mathrm{NUH}$, as previously described, was similar to management at SNEC. ${ }^{6}$ A protocol of intravenous acetazolamide $(500 \mathrm{mg}$, unless the patient had sulfonamide allergy or other contraindications) and eye drops, including topical pilocarpine (4\%), timolol $(0.5 \%)$, brimonidine $(0.1 \%)$, and prednisolone acetate $(1 \%)$ or betamethasone $(0.1 \%)$, were instituted. Patients were reviewed 1 to 2 hours later and immediate LPI with sequential Argon and Nd:YAG laser was performed if the cornea was clear. ${ }^{6}$

Resolution of APAC was defined as IOP $<21 \mathrm{mmHg}$ with resolution of acute symptoms. If the IOP remained elevated $(>21 \mathrm{mmHg})$ with persistent corneal haze despite 2 hours of treatment, the management strategies at the two centers diverged. At NUH, ALPI was performed with settings of $500 \mathrm{~mW}$ power, $100 \mu \mathrm{m}$ spot diameter, and $200 \mathrm{~ms}$ duration, followed by a definitive LPI once corneal clarity improved. At SNEC, 
a trial of systemic medications, such as intravenous mannitol, or further acetazolamide was implemented. The patient charts were reviewed 1-year after APAC, and the development of raised IOP after aborting the attack was recorded and compared between centers. The cup-to-disc ratio and IOP were reviewed together with serial visual field tests to determine the incidence of progression to GON within 1-year of the attack.

\section{Statistical analysis}

All analyses were performed using SPSS version 20 (IBM Corporation, Armonk, NY, USA) and R version 2.14.0 (R Foundation for Statistical Computing, Vienna, Austria). Mean with standard deviation (SD) were calculated for continuous variables and frequency with percentage (\%) were tabulated for categorical variables.

Differences in distribution of continuous variables between SNEC and NUH were assessed using Mann-Whitney $U$-tests. Associations between categorical variables and hospitals (SNEC and NUH) were assessed using Fisher's exact tests. Online calculators developed by Daniel Soper and Richard Lowry were used for the $3 \times 2$ and $4 \times 2$ Fisher's exact tests, respectively. ${ }^{12,13}$

For all tests, $P$-values $<0.05$ were considered statistically significant.

\section{Results}

A total of 92 patients who presented to the two centers with APAC were studied. The demographic features of the patients are summarized in Table 1. Values are expressed as means $\pm \mathrm{SD}$. Across both centers, the majority of patients were Chinese $(89.1 \%)$ and female $(70.7 \%)$, and the mean age was $61.5 \pm 9.7$ years. The mean IOP at presentation was $58.2 \pm 11.6 \mathrm{mmHg}$ and the majority of patients $(80.9 \%)$ presented within 3 days

Table I Demographic characteristics of all subjects who presented with APAC

\begin{tabular}{lllll}
\hline Demographics & $\begin{array}{l}\text { All } \\
(\mathbf{n}=\mathbf{9 2})\end{array}$ & $\begin{array}{l}\text { SNEC } \\
(\mathbf{n}=\mathbf{5 2})\end{array}$ & $\begin{array}{l}\text { NUH } \\
(\mathbf{n}=\mathbf{4 0})\end{array}$ & P-value \\
\hline $\begin{array}{l}\text { Age (years)* } \\
\text { Gender }\end{array}$ & $61.5(9.70)$ & $62.58(8.50)$ & $60.10(11.0 \mathrm{I})$ & 0.157 \\
$\quad \begin{array}{l}\text { Female } \\
\text { Race }\end{array}$ & $65(70.7)$ & $36(69.2)$ & $29 \mathrm{c}(72.5)$ & 0.819 \\
$\quad \begin{array}{ll}\text { Chinese } \\
\text { Malay }\end{array}$ & $82(89.1)$ & $45(86.5)$ & $37(92.5)$ & 0.845 \\
$\quad$ & $9(9.8)$ & $6(11.5)$ & $3(7.5)$ & \\
Others & $\mathrm{I}(1.1)$ & $\mathrm{I}(1.9)$ & $0(0.0)$ & \\
\hline
\end{tabular}

Notes: Data presented are mean (standard deviation) or frequency (percentage), where appropriate; *Mann-Whitney U-test; ${ }^{\circledR}$ Fisher's exact test.

Abbreviations: NUH, National University Hospital; SNEC, Singapore National Eye Centre. of having symptoms. The mean follow up duration was $18.8 \pm 14$ months.

More patients who attended NUH presented within 3 days of the onset of symptoms, compared to those at SNEC, and this difference was significant $(P=0.037)$. There were no significant differences in the presenting VA $(P=0.59)$, IOP $(P=0.97)$, time to perform LPI $(P=0.30)$, or the duration to break the attack $(P=0.11)$. These data are summarized in Table 2.

The differences in treatment between both centers were evaluated (Table 3 ). In both groups, the majority of patients were given intravenous diamox (98.1\% and $95.0 \%$ at SNEC and NUH, respectively). More patients were given oral diamox in SNEC than NUH $(P=0.001)$. Intravenous mannitol was given to $32.7 \%$ of patients in SNEC, whereas it was used in only one patient in NUH. In contrast, ALPI was performed on $30.0 \%$ of patients at NUH, compared to none at SNEC. The mean time to break the attack was $18.2 \pm 32.9$ hours at SNEC and $9.80 \pm 10.6$ hours at NUH, and this difference was not significant $(P=0.11)$. There were no adverse reactions to medical treatment or immediate complications from laser treatment observed in this study.

Of the patients from NUH, 12 had ALPI, compared to 28 patients who did not (Table 4$)$. The initial visual acuity was poorer in the ALPI group $(P=0.018)$, although the initial IOP was not significantly different $(P=0.47)$. The time to LPI was slower in the medically managed group (21.50 hours) than the ALPI group (11.75 hours) but this was not statistically significant $(P=0.516)$. The mean time to break the attack was 8.64 hours in the group that did not have ALPI, and 12.50 hours in the group that had ALPI $(P=0.086)$.

Once the attack was successfully aborted, the IOP in 19 patients from SNEC and 6 patients from NUH increased

Table 2 Comparison of characteristics of the attack eye by hospital

\begin{tabular}{llll}
\hline Characteristics & $\begin{array}{l}\text { SNEC } \\
(\mathbf{n}=\mathbf{5 2})\end{array}$ & $\begin{array}{l}\text { NUH } \\
(\mathbf{n}=\mathbf{4 0})\end{array}$ & P-value \\
\hline $\begin{array}{l}\text { Duration of symptoms } \\
\quad \leq 3 \text { days }\end{array}$ & $37(71.2 \%)$ & $36(90.0 \%)$ & 0.037 \\
$\quad>3$ days & $15(28.8 \%)$ & $4(10.0 \%)$ & \\
$\begin{array}{l}\text { Initial VA of attack eye } \\
\text { Initial IOP }\end{array}$ & $1.05(0.76)$ & $0.98(0.56)$ & 0.590 \\
Time to LPI (hours)* & $55.7(12.1)$ & $54.7(12.2)$ & 0.974 \\
$\begin{array}{l}\text { Duration to break attack } \\
\text { (hours)* }\end{array}$ & $13.2(19.8)$ & $18.6(27.4)$ & 0.302 \\
\hline
\end{tabular}

Notes: Data presented as mean (standard deviation) or frequency (percentage), where appropriate; *Mann-Whitney U-test; ${ }^{\circledR}$ Fisher's exact test.

Abbreviations: IOP, intraocular pressure; LPI, laser peripheral iridotomy; $\mathrm{NUH}$, National University Hospital; SNEC, Singapore National Eye Centre; VA, visual acuity. 
Table 3 Management of patients during acute attack

\begin{tabular}{|c|c|c|c|}
\hline Management & $\begin{array}{l}\text { SNEC } \\
(n=52)\end{array}$ & $\begin{array}{l}\text { NUH } \\
(n=40)\end{array}$ & $P$-value* \\
\hline \multicolumn{4}{|c|}{ Use of IV diamox } \\
\hline No & I (I.9\%) & $2(5.0 \%)$ & 0.578 \\
\hline Yes & $5 \mathrm{I}(98.1 \%)$ & 38 (95.0\%) & \\
\hline \multicolumn{4}{|c|}{ Use of oral diamox } \\
\hline No & 21 (40.4\%) & $3 \mathrm{I}(77.5 \%)$ & 0.001 \\
\hline Yes & 31 (59.6\%) & $9(22.5 \%)$ & \\
\hline \multicolumn{4}{|l|}{ Use of mannitol } \\
\hline No & 35 (67.3\%) & 39 (97.5\%) & $<0.001$ \\
\hline Yes & 17 (32.7\%) & I (2.6\%) & \\
\hline \multicolumn{4}{|l|}{ Iridoplasty done } \\
\hline No & $52(100.0 \%)$ & $28(70.0 \%)$ & $<0.001$ \\
\hline Yes & $0(0.0 \%)$ & $12(30.0 \%)$ & \\
\hline
\end{tabular}

Notes: Data presented are frequency (percentage); *Fisher's exact test. Abbreviations: IV, intravenous; NUH, National University Hospital; SNEC, Singapore National Eye Centre.

$(P=0.032)$ within 1-year (Table 5). Of these, GON developed in 13 patients from SNEC and all six patients from NUH. All patients who developed raised IOP, including patients with raised IOP after APAC and patients who developed PACG with suboptimal IOP, were first treated with topical ocular hypotensive medication.

The indication for cataract surgery in both groups was a visually significant cataract. Seventeen patients from SNEC and 13 patients from NUH had cataract surgery and there was no significant difference in the time until surgery was performed $(P=0.700)$. In the group of patients who developed GON, one patient from SNEC and seven from NUH underwent combined phacoemulsification and trabeculectomy with mitomycin-C. From NUH, one patient had trabeculectomy with mitomycin-C alone, and another had combined cataract surgery with the insertion of a glaucoma drainage device.

At final review, the mean IOP of the APAC eye was $14.8 \pm 4.3 \mathrm{mmHg}$ from SNEC and $13.4 \pm 3.0 \mathrm{mmHg}$ from NUH. There was no significant difference in VA $(P=0.491)$ or IOP $(P=0.224)$ between both groups.

\section{Discussion}

This is the first study to compare the visual outcomes following APAC with different management approaches within an Asian population with similar demographic features. In contrast to Caucasians, studies suggest poorer outcomes in Asians after APAC, with more Asians developing chronic angle closure glaucoma and eventually requiring surgical intervention. ${ }^{14}$ Studies have suggested that APAC attacks damage the drainage angle and cause irreversible ischemic changes to the optic nerve head, and
Table 4 Comparison of the management of subjects from NUH by iridoplasty status

\begin{tabular}{|c|c|c|c|}
\hline \multirow[t]{2}{*}{ Characteristics } & \multicolumn{2}{|c|}{ Iridoplasty done } & \multirow[t]{2}{*}{$P$-value } \\
\hline & No $(n=28)$ & Yes $(n=12)$ & \\
\hline Initial VA of attack eye & $0.8 \mathrm{I}(0.4 \mathrm{I})$ & $1.37(0.66)$ & 0.018 \\
\hline Initial IOP & $53.6(11.2)$ & $57.2(14.6)$ & 0.469 \\
\hline \multicolumn{4}{|l|}{ Duration of symptoms } \\
\hline$\leq 3$ days & 26 (92.9\%) & $10(83.3 \%)$ & 0.570 \\
\hline$>3$ days & $2(7.1 \%)$ & $2(16.7 \%)$ & \\
\hline \multicolumn{4}{|l|}{ Use of IV diamox } \\
\hline No & $2(7.1 \%)$ & $0(0.0 \%)$ & 1.000 \\
\hline Yes & $26(92.9 \%)$ & $12(100 \%)$ & \\
\hline \multicolumn{4}{|l|}{ Use of oral diamox } \\
\hline No & $21(75.0 \%)$ & $10(83.3 \%)$ & 0.697 \\
\hline Yes & 7 (25.0\%) & $2(16.7 \%)$ & \\
\hline \multicolumn{4}{|l|}{ Use of mannitol } \\
\hline No & 27 (96.4\%) & $12(100.0 \%)$ & 1.000 \\
\hline Yes & I $(3.6 \%)$ & $0(0.0 \%)$ & \\
\hline Time to LPI (hours) & $21.5(32.0)$ & II.8 (8.4) & 0.516 \\
\hline $\begin{array}{l}\text { Duration to break attack } \\
\text { (hours) }\end{array}$ & $8.6(I I .2)$ & $12.5(9.1)$ & 0.086 \\
\hline \multicolumn{4}{|l|}{ Time to IOP rise } \\
\hline No rise & $21(75.0 \%)$ & $10(83.3 \%)$ & 1.000 \\
\hline$\leq 12$ months & $4(14.3 \%)$ & I (8.3\%) & \\
\hline$>12$ months & $3(10.7 \%)$ & I (8.3\%) & \\
\hline \multicolumn{4}{|c|}{ Development of raised IOP } \\
\hline No & $23(71.4 \%)$ & II (83.3\%) & 0.648 \\
\hline Yes & $5(28.6 \%)$ & I (I6.7\%) & \\
\hline \multicolumn{4}{|l|}{ Development of GON } \\
\hline No & $23(7 \mathrm{I} .4 \%)$ & II (83.3\%) & 0.648 \\
\hline Yes & $5(28.6 \%)$ & I (16.7\%) & \\
\hline
\end{tabular}

Notes: Data presented as mean (standard deviation) or frequency (percentage), where appropriate; Mann-Whitney U-test or Fisher's exact test is used, where appropriate.

Abbreviations: GON, glaucomatous optic neuropathy; IOP, intraocular pressure; IV, intravenous; LPI, laser peripheral iridotomy; NUH, National University Hospital; SNEC, Singapore National Eye Centre; VA, visual acuity.

Table 5 One year outcome after acute primary angle closure

\begin{tabular}{|c|c|c|c|}
\hline Outcome & SNEC $(n=52)$ & NUH $(n=40)$ & $P$-value* \\
\hline \multicolumn{4}{|c|}{ Development of raised IOP\$ } \\
\hline No & $33(63.5 \%)$ & $34(77.5 \%)$ & 0.032 \\
\hline Yes & $19(36.5 \%)$ & $6(22.5 \%)$ & \\
\hline \multicolumn{4}{|l|}{ Cataract surgery } \\
\hline No & $35(67.3 \%)$ & 27 (67.5\%) & 1.000 \\
\hline Yes & $17(32.7 \%)$ & $13(32.5 \%)$ & \\
\hline $\begin{array}{l}\text { Time to cataract } \\
\text { surgery }\end{array}$ & & & 0.700 \\
\hline$\leq 3$ months & $10(58.8 \%)$ & $5(45.5 \%)$ & \\
\hline$>3$ months & $7(4 \mid .2 \%)$ & $6(54.5 \%)$ & \\
\hline \multicolumn{4}{|c|}{ Development of GON } \\
\hline No & $39(75.0 \%)$ & $34(75.0 \%)$ & 0.303 \\
\hline Yes & $13(25.0 \%)$ & $6(25.0 \%)$ & \\
\hline \multicolumn{4}{|c|}{ Time to glaucoma surgery } \\
\hline No surgery & $51(98.1 \%)$ & $32(80.0 \%)$ & 0.011 \\
\hline$\leq 12$ months & $\mathrm{I}(1.9 \%)$ & $5(12.5 \%)$ & \\
\hline$>12$ months & $0(0.0 \%)$ & $3(7.5 \%)$ & \\
\hline
\end{tabular}

Notes: Data presented as frequency (percentage); ${ }^{* P}$-value based on Fisher's exact

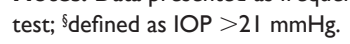

Abbreviations: GON, glaucomatous optic neuropathy; IOP, intraocular pressure; NUH, National University Hospital; SNEC, Singapore National Eye Centre. 
prompt therapy to abort the attack could limit the insult to these ocular structures. ${ }^{1-3,9}$ Whether or not more rapid lowering of IOP with a paracentesis is superior to our more gradual reductions in the high risk Asian population has yet to be proven. It is generally considered only in recalcitrant cases, considering the technical difficulties and potential complications.

The duration from the onset to termination of APAC exposes the optic nerve to high intraocular pressures and is expected to influence outcomes in eyes with APAC. Furthermore, a study has shown that more than $50 \%$ of patients fail to respond to medical treatment during the acute attack, and patients with delayed presentation have a relative risk of 2.78 times for developing chronic glaucoma. ${ }^{10}$ This suggests that a more aggressive approach with rapid IOP lowering should be advocated.

In our study, the duration of symptoms was classified into greater or less than 3 days, as a previous study showed that a mean presentation within 3 days of symptoms was associated with good visual outcome. ${ }^{6} \mathrm{We}$ found that patients who presented to SNEC had a longer duration of symptoms prior to presentation ( $P=0.037)$, and this was associated with the development of raised IOP that was statistically significant $(P=0.032)$. However, we found that there was no significant difference in final VA or the development of GON between the two groups. Our results complement the findings of a recent study involving 42 eyes of 41 Singapore patients, which identified that delayed presentation is a risk factor for future PACG. ${ }^{6}$ Although our study duration may be too short to demonstrate a difference in GON development, it is likely that raised IOP may preclude eventual progression to GON. In contrast, another study conducted in Singapore of 90 patients found that delayed presentation was not associated with the late development of chronic IOP rise or the prevalence of PACG. ${ }^{9}$ However, this study did not exclude patients with preexisting glaucoma, which may confound the results. A follow up study on our group of patients would be informative.

ALPI has been shown to dramatically reduce IOP and permit corneal clearing for LPI to be performed. ${ }^{8,15}$ In cases of APAC that are unresponsive to medical treatment, and LPI is prevented by corneal haze, ALPI has been shown to be effective in all cases for rapid and significant IOP reduction. ${ }^{16}$ However, this approach has not shown to significantly affect outcomes, and exposes patients to procedural risks, such as corneal decompensation and iris and lens damage. The patients with recalcitrant attacks were selected for ALPI after failed medical therapy, and hence had a longer mean duration to break the attack compared to patients who were treated medically. Increased inflammation from a combination of ALPI and LPI compared to LPI only may also be contributory. However, the time to LPI was faster in the ALPI group, which may be important during the outof-hours setting when manpower is scarce. Of note, none of the patients in our study developed any complications after ALPI.

A comparison between the use of ALPI and systemic medications (acetazolamide \pm mannitol) found that ALPI rapidly lowered IOP compared to systemic medication in the first 2 hours of treatment, following which there was no difference in mean final IOP or a need for glaucoma medications. ${ }^{17}$ Medical therapy alone was shown to successfully abort an APAC attack within 12 hours in $76.2 \%$ of patients and within 24 hours in $89.2 \%$ of patients. ${ }^{18}$

In our study, patients treated medically had a longer interval to LPI compared to the ALPI group. A possible explanation for this may be that patients who were treated medically during the out-of-hours setting had successful IOP lowering that allowed for LPI to be delayed until the next morning. However, in patients with failed medical therapy and persistently raised IOP, ALPI would provide an important window of corneal clarity when the LPI may be performed. In this case, LPI would have to be completed with relative urgency.

Although our patient numbers may be too small to detect statistically significant differences in the time taken to break an attack of APAC between the two centers $(P=0.11)$, it may be considered clinically relevant as it entails prompt symptomatic relief for patients. This must be balanced against the availability of an experienced ophthalmologist who can competently perform ALPI at whatever time the APAC patient presents. Both approaches seem acceptable options and may be chosen by personal preference.

There are several limitations to our study design. This is a retrospective study with a small sample size and a short 1-year follow up duration. Despite a protocol for the initial management of APAC, patients are managed at the discretion of the onsite ophthalmologist, and thresholds for another treatment strategy may differ accordingly. Disc evaluation in the acute setting, as well as PACG diagnosis, is difficult in the context of a hazy cornea. We evaluated the optic disc once the attack was broken and the cornea was clearer. Therefore, the patients are promptly referred to the glaucoma services for further evaluation and subsequent management in the respective hospitals. The diagnoses of PACG and conversion to GON were made by glaucoma-trained 
consultant ophthalmologists. Furthermore, the follow up care and documentation by different ophthalmologists was not standardized. While a similar time to cataract surgery existed in each group, it is difficult to extract the effect of this on the final outcomes as our study was inadequately powered to detect this.

In conclusion, treatment strategies in both centers were effective in aborting an APAC attack regardless of the duration of symptoms prior to treatment. However, the development of raised IOP, but not GON, was associated with a longer period of attack. Our findings validate the sense of urgency when dealing with patients with APAC as the length of attack may entail poorer outcomes. Prospective studies with longer follow up periods are needed to determine if patients with raised IOP are at risk of eventually developing GON.

\section{Disclosure}

Dr T. Aung has received research funding, travel support and honoraria from Carl Zeiss Meditec. Dr Perera has received honoraria from Carl Zeiss Meditec and Allergan. The authors report no other conflicts of interest in this work.

\section{References}

1. Sng CC, See JS, Ngo CS, et al. Changes in retinal nerve fibre layer, optic nerve head morphology, and visual field after acute primary angle closure. Eye (Lond). 2011;25(5):619-625.

2. Tsai JC, Lin PW, Teng MC, Lai IC. Longitudinal changes in retinal nerve fiber layer thickness after acute primary angle closure measured with optical coherence tomography. Invest Ophthalmol Vis Sci. 2007; 48(4):1659-1664.

3. Aung T, Husain R, Gazzard G, et al. Changes in retinal nerve fiber layer thickness after acute primary angle closure. Ophthalmology. 2004; 111(8):1475-1479.

4. Seah SK, Foster PJ, Chew PT, et al. Incidence of acute primary angle-closure glaucoma in Singapore. An island-wide survey. Arch Ophthalmol. 1997;115(11):1436-1440.
5. Aung T, Ang LP, Chan SP, Chew PT. Acute primary angle-closure: long-term intraocular pressure outcome in Asian eyes. Am JOphthalmol. 2001;131(1):7-12.

6. Tan AM, Loon SC, Chew PT. Outcomes following acute primary angle closure in an Asian population. Clin Experiment Ophthalmol. 2009; 37(5):467-472.

7. Saw SM, Gazzard G, Friedman DS. Interventions for angle-closure glaucoma: an evidence-based update. Ophthalmology. 2003; 110(10):1869-1878.

8. Lam DS, Lai JS, Tham CC, Chua JK, Poon AS. Argon laser peripheral iridoplasty versus conventional systemic medical therapy in treatment of acute primary angle-closure glaucoma : a prospective, randomized, controlled trial. Ophthalmology. 2002;109(9):1591-1596.

9. Aung T, Friedman DS, Chew PT, et al. Long-term outcomes in Asians after acute primary angle closure. Ophthalmology. 2004; 111(8):1464-1469.

10. Wong JS, Chew PT, Alsagoff Z, Poh K. Clinical course and outcome of primary acute angle-closure glaucoma in Singapore. Singapore Med J. 1997;38(1):16-18.

11. Lee JW, Lee JH, Lee KW. Prognostic factors for the success of laser iridotomy for acute primary angle closure glaucoma. Korean $J$ Ophthalmol. 2009;23(4):286-290.

12. Fisher exact probability test for a $2 \times 3$ contingency table [webpage on the Internet]. Soper D; 2006. Available from: http://www.danielsoper. $\mathrm{com} /$ statcalc3/calc. aspx?id=58. Accessed March 20, 2013.

13. Fisher Exact Probability Test: $2 \times 4$ [webpage on the Internet]. City: Lowry R; 2001. Available from: http://vassarstats.net/fisher $2 \times 4 . h t m l$. Accessed March 20, 2013.

14. Fleck BW, Wright E, Fairley EA. A randomised prospective comparison of operative peripheral iridectomy and Nd:YAG laser iridotomy treatment of acute angle closure glaucoma: 3 year visual acuity and intraocular pressure control outcome. Br J Ophthalmol. 1997; 81(10):884-888.

15. Lam DS, Lai JS, Tham CC. Immediate argon laser peripheral iridoplasty as treatment for acute attack of primary angle-closure glaucoma: a preliminary study. Ophthalmology. 1998;105(12):2231-2236.

16. Lim AS, Tan A, Chew P, et al. Laser iridoplasty in the treatment of severe acute angle closure glaucoma. Int Ophthalmol. 1993;17(1):33-36.

17. Lai JS, Tham CC, Chua JK, et al. To compare argon laser peripheral iridoplasty (ALPI) against systemic medications in treatment of acute primary angle-closure: mid-term results. Eye (Lond). 2006; 20(3):309-314.

18. Ramli N, Chai SM, Tan GS, et al. Efficacy of medical therapy in the initial management of acute primary angle closure in Asians. Eye (Lond). 2010;24(10):1599-1602.
Clinical Ophthalmology

\section{Publish your work in this journal}

Clinical Ophthalmology is an international, peer-reviewed journal covering all subspecialties within ophthalmology. Key topics include: Optometry; Visual science; Pharmacology and drug therapy in eye diseases; Basic Sciences; Primary and Secondary eye care; Patient Safety and Quality of Care Improvements. This journal is indexed on Submit your manuscript here: http://www.dovepress.com/clinical-ophthalmology-journal
Dovepress

PubMed Central and CAS, and is the official journal of The Society of Clinical Ophthalmology (SCO). The manuscript management system is completely online and includes a very quick and fair peer-review system, which is all easy to use. Visit http://www.dovepress.com/ testimonials.php to read real quotes from published authors. 